\title{
Descomposición de hojarasca en seis arroyos de Bosque Mesófilo de Montaña en la cuenca alta del río La Antigua, Veracruz, México
}

\author{
Manuel R. Astudillo ${ }^{1}$, Alonso Ramírez ${ }^{2}$, Rodolfo Novelo-Gutiérrez ${ }^{3}$ \& Gabriela Vázquez ${ }^{4}$ \\ 1. Doctorado en Ciencias, Instituto de Ecología, A.C., Xalapa, Veracruz, México; bosque_nocturno@hotmail.com \\ 2. Departamento de Ciencias Ambientales, Universidad de Puerto Rico, San Juan, Puerto Rico; \\ aramirez@ramirezlab.net \\ 3. Red de Biodiversidad y Sistemática, Instituto de Ecología, A.C., Xalapa, Veracruz, México; \\ rodolfo.novelo@inecol.mx \\ 4. Red de Ecología Funcional, Instituto de Ecología, A.C., Xalapa, Veracruz, México; gabriela.vazquez@inecol.mx
}

\author{
Recibido 12-XII-2013. Corregido 20-I-2014. Aceptado 13-II-2014.
}

\begin{abstract}
Leaf litter decomposition in six Cloud Forest streams of the upper La Antigua watershed, Veracruz, Mexico. Leaf litter decomposition is an important stream ecosystem process. To understand factors controlling leaf decomposition in cloud forest in Mexico, we incubated leaf packs in different streams along a land use cover gradient for 35 days during the dry and wet seasons. We assessed relations between leaf decomposition rates $(k)$, stream physicochemistry, and macroinvertebrates colonizing leaf packs. Physicochemical parameters showed a clear seasonal difference at all study streams. Leaves were colonized by collector-gatherer insects, followed by shredders. Assessment of factors related to $k$ indicated that only forest cover was negatively related to leaf decomposition rates. Thus stream physicochemistry and seasonality had no impact on decomposition rates. We concluded that leaf litter decomposition at our study streams is a stable process over the year. However, it is possible that this stability is the result of factors regulating decomposition during the different seasons and streams. Rev. Biol. Trop. 62 (Suppl. 2): 111-127. Epub 2014 April 01.
\end{abstract}

Key words: leaf breakdown, aquatic insects, forest cover, functional feeding groups.

En los sistemas acuáticos, el material alóctono como la hojarasca proveniente de la vegetación ribereña, es de gran importancia debido a que en muchos arroyos la sombra del dosel limita la productividad primaria, convirtiendo el material alóctono en la base trófica del ecosistema (Webster \& Benfield, 1986; Benstead, 1996; Wallace, Eggert, Meyer \& Webster, 1997). De este modo, la hojarasca es utilizada como alimento por una gran variedad de organismos y como sustrato por otros (Aldridge, Brookes \& Ganf, 2009). La forma en que los insectos acuáticos aprovechan la hojarasca es variada. Los fragmentadores utilizan la hojarasca como alimento, mientras los recolectores aprovechan las partículas que se acumulan en ésta; los depredadores, por su parte, la utilizan como un sitio para encontrar presas y refugio (Benstead, 1996).

La descomposición de hojarasca es un proceso complejo que depende de factores bióticos y abióticos, tales como las características propias de la hojarasca, las condiciones fisicoquímicas del cuerpo de agua y los organismos que la colonizan y consumen (Graça, 2001; Leroy \& Marks, 2006; Pérez, Menéndez, Larrañaga \& Pozo, 2011; Yoshimura, 2012). Factores a distinta escala (p.ej. concentración de iones, la cobertura vegetal ribereña, el tipo de suelo y el clima) afectan el proceso de descomposición de la hojarasca a la vez que favorecen a los diferentes organismos (i.e. bacterias, hongos, insectos) que pueden aprovecharla para su desarrollo (Casas, Gessner, López \& Descals, 
2011). Otro factor que afecta este proceso es el uso del suelo (Paul, Meyer \& Couch, 2006; Yoshimura, 2012). Este al influir sobre los factores ambientales (i.e. nutrientes, sedimentación) o en la fauna acuática (Sponseller \& Benfield, 2001), afecta de forma indirecta el proceso de descomposición. El efecto del uso del suelo sobre la descomposición puede no reflejarse en la tasa de descomposición pero sí mostrar cambios en los factores que la afectan. En estudios comparativos entre pastizales y bosque, Huryn, Butz-Huryn, Arbuckle \& Tsomides (2002) no encontraron diferencias entre las tasas de descomposición de la hojarasca en estos dos usos del suelo, a pesar de que en los pastizales se registró la mayor concentración de nutrientes y la menor cantidad de fragmentadores, mientras que en el bosque se registró una baja concentración de nutrientes y una mayor cantidad de fragmentadores.

La estacionalidad es otro factor que afecta la descomposición de la hojarasca. Niu \& Dudgeon (2011) encontraron que en arroyos tropicales, la descomposición de la hojarasca en la época seca estuvo influenciada principalmente por las abundancias de recolectores (que pueden incrementar el rompimiento de la hojarasca) y fragmentadores (los cuales se alimentan de ella). En cambio, en la época lluviosa, la variable que más influyó sobre la descomposición fue la descarga, que puede aumentar la fragmentación debido a la acción física del flujo. En otro estudio, Ferreira, Graça, Lima \& Gomes (2006) encontraron resultados similares a los anteriores para la época lluviosa, mientras que en la época de menor precipitación fue más importante el efecto de los fragmentadores.

La mayoría de los estudios sobre descomposición de hojarasca en arroyos, han sido realizados en zonas templadas, mientras que para el trópico el interés en este tema es reciente (i.e. Graça et al., 2001; Biggs, Dunne \& Martinelli, 2004; Boyero, Pearson \& Bastian, 2007; Gonçalves, Rezende, França \& Callisto, 2012). En México es poco lo que se conoce sobre este proceso en arroyos rodeados por bosque mesófilo de montaña. Este tipo de vegetación ha sido catalogado como el más diverso por unidad de área, y sin embargo ha disminuido a casi la mitad de su área desde 1973 debido principalmente a la deforestación (Martínez et al., 2009). Por lo tanto, es importante conocer la biodiversidad y los procesos funcionales, como la descomposición de hojarasca, que se llevan a cabo en los arroyos del bosque mesófilo y cómo están siendo afectados por la deforestación.

El objetivo de este estudio fue analizar la relación entre la descomposición de la hojarasca con variables fisicoquímicas del agua e insectos acuáticos colonizadores, en arroyos con diferente grado de cobertura boscosa en su área de captación, durante las épocas seca y lluviosa. Nuestras hipótesis fueron las siguientes: En los arroyos con una mayor cobertura boscosa, la variabilidad estacional en las características fisicoquímicas será menor en comparación con los arroyos de menor cobertura boscosa. La diversidad de insectos acuáticos colonizadores de la hojarasca será distinta entre las épocas debido a la mayor frecuencia de disturbios (i.e. crecidas) durante la época lluviosa. El porcentaje de cobertura boscosa en el área de captación modificará la tasa de descomposición, la diversidad de insectos colonizadores y la abundancia relativa de los distintos taxa y grupos funcionales debido a los cambios que ocurren en los arroyos conforme disminuye la cobertura boscosa en el área de captación.

\section{MATERIALES Y MÉTODOS}

Área de estudio: La cuenca del río La Antigua está ubicada entre los $19^{\circ} 05^{\prime}$ y $19^{\circ} 34^{\prime} \mathrm{N}$, y entre los $96^{\circ} 06^{\prime}$ y $97^{\circ} 16^{\prime} \mathrm{W}$, con un área aproximada de $2623 \mathrm{~km}^{2}$ (Martínez et al., 2009). La parte alta de la cuenca, que se ubica en los estados de Veracruz y Puebla, se compone principalmente de arroyos que van de primero a cuarto orden y que confluyen al río Los Pescados, el cual desemboca al Golfo de México (Navarrete-Vázquez, 2008). En su parte alta, la cuenca del río La Antigua presenta una elevación entre los 600 y $2500 \mathrm{~m}$ de altitud. Los valores promedios anuales de temperatura y precipitación para la parte baja del rango 
altitudinal anterior son de $22^{\circ} \mathrm{C}$ y $1300 \mathrm{~mm}$, mientras la parte alta muestra valores de $12^{\circ} \mathrm{C}$ y $2000 \mathrm{~mm}$ (Muñoz-Villers \& López-Blanco, 2008). Esta cuenca es de gran importancia para la agricultura regional, ya que en ella se cultiva café y maíz, entre otros; y es la fuente principal de agua para las ciudades de Coatepec y Xalapa, capital del estado de Veracruz (Pereyra, Cruz \& Pérez, 2011).

El tipo de vegetación presente en la zona es principalmente bosque mesófilo de montaña (BMM), el cual se encuentra entre los 1200 y 2100 metros de altitud en lomeríos con suelos volcánicos y es el tipo más diverso por unidad de área en México (García-Franco, Castillo-Campos, Mehltreter, Martínez \& Vázquez, 2008). El BMM en el área de estudio se encuentra en forma de remanentes inmersos en un paisaje compuesto por una matriz de campos agrícolas, potreros, cafetales y asentamientos humanos (Williams-Linera, 1993). El BMM presenta gran variación en la estructura y composición vegetal entre sus distintos fragmentos debido a la gran variación topográfica y microambiental (García-Franco et al., 2008), lo cual ha permitido la coexistencia de flora templada y neotropical (Williams-Linera et al., 2002). Algunos de los árboles más comunes son Liquidambar styraciflua, Quercus xalapensis, $Q$. leiophylla, entre otros (Williams-Linera, Manson \& Isunza, 2002). Como sustrato para el experimento de descomposición, se seleccionaron hojas de haya o álamo, Platanus mexicana, que es una especie común en el área de estudio. Esta es una especie caducifolia que llega a medir de 15 a $25 \mathrm{~m}$, con un nivel máximo de $40 \mathrm{~m}$ de alto, con diámetro a la altura del pecho de 0.8 a $1 \mathrm{~m}$ y se establece principalmente a lo largo de arroyos y ríos (Martínez, 1994).

Selección de sitios: Se seleccionaron seis arroyos a lo largo de un gradiente de cobertura boscosa en su área de captación (Cuadro 1). El porcentaje de cobertura boscosa se obtuvo con los programas ArcGis (Versión 10), Erdas (Versión 8.7) y eCognition Developer (versión 8) (Landgrave, datos sin publicar) utilizando ortofotos rectificadas de la región a escala 1:50 000 tomadas en 2004 con resolución digital de $1 \mathrm{~m}$ por pixel (INEGI, 2004). Los arroyos seleccionados se encuentran entre los 1595 y $1991 \mathrm{msnm}$ y van de primero a tercer orden (Cuadro 1). En cada arroyo se seleccionó un tramo de $100 \mathrm{~m}$ donde se midieron variables fisicoquímicas in situ, se tomaron muestras de agua para las épocas seca (abril-mayo 2011) y lluviosa (agosto-octubre 2011) para su posterior análisis en laboratorio, y se ubicaron los grupos de hojas. El sitio de estudio en Chivizcoyo se agregó durante la época lluviosa, por lo que no se midió la descomposición de la hojarasca durante la época seca.

Variables fisicoquímicas del agua: Las medidas in situ que se realizaron fueron el $\mathrm{pH}$ con un potenciómetro (Barnant Mod. 20), y la temperatura, oxígeno disuelto (OD) y conductividad (Cond) utilizando un multiparamétrico (YSI 85). Estas medidas fueron tomadas por triplicado para pozas y rápidos. Para calcular

CUADRO 1

Características y uso del suelo (\%) para las áreas de captación de los arroyos seleccionados para el estudio

TABLE 1

Characteristics and land use (\%) of the stream catchment areas selected for the study

\begin{tabular}{lcccccc} 
& El Chorrito & Xico Viejo & Monte Grande & Tlalchi & Piedra Blanca & Chivizcoyo \\
Orden & 1 & 3 & 2 & 3 & 3 & 1 \\
Elevación (m) & 1991 & 1744 & 1692 & 1595 & 1621 & 1801 \\
Bosques & 15.82 & 22.9 & 29.82 & 39.08 & 59 & 80 \\
Pastizales & 77.86 & 69.8 & 61.98 & 55.23 & 39.95 & 16 \\
Agricultura & 1.58 & 2.82 & 6.11 & 2.46 & 1.05 & 0 \\
Urbana & 4.75 & 4.48 & 2.09 & 3.23 & 0 & 3 \\
\hline
\end{tabular}


la descarga, en dos transectos transversales se midieron la profundidad (utilizando un metro) y la velocidad instantánea (usando un flujómetro Probe 101FP201). La descarga $(Q)$ se calculó con la fórmula $Q=A v$, siendo $A$ el área de la sección transversal y $v$ la velocidad promedio (Hauer \& Lamberti, 2007). En cada arroyo se tomaron dos muestras de agua, que fueron refrigeradas para procesarlas en el laboratorio utilizando los métodos de APHA (1998) en un periodo menor a $48 \mathrm{hrs}$. Se determinaron las siguientes variables: amonio $\left(\mathrm{NH}_{4}^{+}\right.$, método Nessler), nitratos $\left(\mathrm{NO}_{3}^{-}\right.$, método colorimétrico), nitritos $\left(\mathrm{NO}_{2}^{-}\right.$, método colorimétrico), fósforo total (PT, digestión con persulfato y colorimetría con el método del ácido ascórbico), fósforo reactivo $\left(\mathrm{PO}_{4}{ }^{3-}\right.$, colorimetría con el método del ácido ascórbico) y sólidos suspendidos totales (SST, método gravimétrico).

Análisis estadístico: Para detectar patrones espaciales y/o temporales de las variables fisicoquímicas entre los arroyos, se realizó un análisis de componentes principales (ACP) utilizando el programa PC-ORD (versión 5.0). Todas las variables fueron transformadas con $\log _{10}(\mathrm{x}+1)$ para ser normalizadas, y fueron centradas y estandarizadas en el ACP. Los datos de $\mathrm{pH}$ no fueron transformados debido a que presentaron una distribución normal.

Descomposición de hojarasca: La tasa de descomposición se obtuvo siguiendo el método propuesto por Barlocher (2005). Se recolectaron hojas de $P$. mexicana y fueron secadas a temperatura ambiente ocho días antes de ser colocadas en los arroyos. Se hicieron grupos de cuatro hojas con un peso promedio de $2.5 \mathrm{~g}$, los cuales se pesaron y se empaquetaron en bolsas de red plástica de 20x20 cm con abertura de malla de $5 \mathrm{~mm}$. En cada arroyo se colocaron tres grupos de cinco paquetes de hojarasca cada uno (15 paquetes en total); cada grupo de paquetes se colocó en un remanso del arroyo distribuidos en un tramo de aproximadamente $100 \mathrm{~m}$. Además, cuatro paquetes fueron transportados a los arroyos y regresados al laboratorio sin sumergirlos para estimar el peso seco perdido por los paquetes debido al transporte y la manipulación. Los paquetes de hojarasca se recolectaron a los 7, 14, 21, 28 y 35 días después de iniciado el experimento.

Cada vez que se recogieron los paquetes de hojarasca se colocaron en bolsas de plástico con cierre hermético y se refrigeraron hasta su procesamiento en el laboratorio. Las hojas se lavaron con agua para eliminar el sedimento y obtener los insectos acuáticos asociados. Las hojas lavadas fueron puestas en un horno durante $72 \mathrm{hrs}$ a $40^{\circ} \mathrm{C}$ para después ser pesadas. Posteriormente, las hojas de cada paquete se trituraron y se tomó un gramo del triturado para ser quemado en una mufla a $500^{\circ} \mathrm{C}$ por $2 \mathrm{hrs}$. Se calculó el peso seco libre de ceniza inicial de cada paquete y el porcentaje de peso seco libre de cenizas restante en el paquete.

Dichos porcentajes se transformaron utilizando logaritmo natural para calcular la tasa de descomposición diaria $(k)$ mediante regresiones lineales. Con el fin de comparar las tasas de descomposición entre las épocas seca y lluviosa, utilizando las tasas de descomposición de cada arroyo por época cuyas regresiones lineales fueron significativas, se realizó la prueba de Kruskal-Wallis. Se seleccionó esta prueba ya que las tasas de descomposición para las épocas no presentaron una distribución normal. La misma prueba se realizó utilizando el porcentaje de peso seco libre de ceniza restante a los 28 días de sumergidos los paquetes. Se eligió hacer la comparación a los 28 días debido a que ésta fue la última fecha en que se obtuvieron paquetes de hojarasca, sin que hubiera pérdidas de los mismos, para todos los arroyos y en ambas épocas.

Insectos acuáticos: Los insectos acuáticos hallados en cada paquete de hojarasca se colocaron en envases plásticos con etanol al $96 \%$. Los organismos fueron determinados y cuantificados al nivel taxonómico más bajo posible, con excepción de Chironomidae (Diptera) debido a que las claves de identificación a nivel de género son aun limitadas. Los taxa fueron asignados a grupos funcionales de acuerdo a lo propuesto por Merritt \& Cummins 
(1996) y Cummins, Merrit \& Andrade (2005). Debido a que no se recuperó el mismo número de paquetes para cada arroyo y fecha de colecta (a causa de la intervención antrópica o arrastre por el arroyo), se sumaron las abundancias de los insectos de todas las fechas de recolecta y se trabajó con las abundancias promedio del total de insectos acuáticos colonizadores de los paquetes de hojarasca para cada arroyo. Con el fin de observar la similitud de los arroyos de acuerdo a la composición de los insectos colonizadores de los paquetes de hojarasca, se hicieron análisis de agrupamiento con los arroyos para cada época utilizando el índice de Jaccard con el programa PC-ORD (versión 5.0).

Relación entre la tasa de descomposición, porcentaje de bosque, los insectos acuáticos y la fisicoquímica del agua: Finalmente, se realizó una regresión múltiple por pasos (stepwise) para determinar la relación entre la tasa de descomposición de la hojarasca y las variables fisicoquímicas, los insectos fragmentadores, los no fragmentadores (raspadores, recolectores y depredadores) y el porcentaje de cobertura boscosa. De las variables fisicoquímicas medidas solo se utilizaron las que más aportaron al primer eje del $\mathrm{ACP}(Q$, $\mathrm{PT}$, temperatura y $\mathrm{NH}_{4}^{+}$), con excepción del oxígeno, ya que no fue posible normalizarlo después de remover los datos de los arroyos para los que los valores de $k$ no fueron significativos. La misma regresión por pasos se realizó utilizando el porcentaje de peso seco libre de cenizas después de 28 días en vez de la tasa de descomposición. Las regresiones fueron realizadas utilizando el programa Statistica (versión 7.1).

\section{RESULTADOS}

Variables fisicoquímicas del agua: $\mathrm{El} \mathrm{pH}$ presentó valores cercanos a la neutralidad en todos los arroyos. El valor más ácido se registró en Monte Grande en época seca (5.89) y el valor más básico se midió en Piedra Blanca en época seca (7.49) (Cuadro 2). La temperatura más baja se registró en el arroyo El Chorrito en época seca, mientras el mayor valor lo presentó Xico Viejo en época seca $\left(20.65^{\circ} \mathrm{C}\right)$. Los valores de OD para cada arroyo fueron mayores en la época lluviosa, con excepción de El Chorrito que presentó valores muy similares en ambas épocas. El arroyo con más OD fue Xico Viejo en época lluviosa, mientras el de menor OD fue Monte Grande en época seca. Al comparar la conductividad de cada arroyo en las dos épocas, éstos presentaron mayores valores en la época seca. La menor concentración de $\mathrm{NH}_{4}{ }^{+}$se registró en El Chorrito en época seca y la mayor en el arroyo de Tlalchi en la misma época. El PT presentó los valores más bajos en la época lluviosa en todos los arroyos. $\mathrm{La}$ concentración de $\mathrm{NO}_{3}{ }^{-}+\mathrm{NO}_{2}^{-}$fue mayor en la época lluviosa para casi todos los arroyos, exceptuando el arroyo de Monte Grande. La descarga fue mayor para cada arroyo en la época lluviosa.

Análisis estadístico: Los dos primeros ejes del ACP explicaron el $62.3 \%$ de la varianza. El eje 1 explicó el $36.2 \%$ y reveló un gradiente de temporalidad entre los arroyos, asociados a la descarga, al $\mathrm{OD}, \operatorname{los} \mathrm{NO}_{3}^{-}+$ $\mathrm{NO}_{2}^{-}$, el PT, el $\mathrm{NH}_{4}^{+}$y la temperatura. Sobre el eje 1 en el extremo izquierdo de la ordenación, se agruparon los arroyos de Xico Viejo, Tlalchi, el Chorrito y Piedra Blanca en la época lluviosa, con los mayores valores de descarga, y las mayores concentraciones de $\mathrm{OD} \mathrm{y} \mathrm{NO}_{3}{ }^{-+}$ $\mathrm{NO}_{2}^{-}$. En el extremo derecho de la ordenación se ubicaron los arroyos de Chivizcoyo, Xico Viejo, Tlalchi y Piedra Blanca en la época seca, con las mayores concentraciones de $\mathrm{PT}, \mathrm{NH}_{4}{ }^{+}$ y temperatura (Fig. 1). El Chorrito en época seca fue la excepción, al estar más cercano a los arroyos de la época lluviosa. También se observa que el arroyo de Monte Grande se diferenció de los demás sitios en ambas épocas al presentar los menores valores de $\mathrm{pH}$, conductividad y $\mathrm{PO}_{4}{ }^{3-}$. El eje 2 explicó el $26.1 \%$ de la varianza total y mostró un gradiente de mineralización al estar asociado principalmente a la conductividad y al $\mathrm{pH}$. En la época lluviosa los arroyos se ordenaron a lo largo del eje 2 del Chorrito, Xico Viejo, Tlalchi a Piedra Blanca, 


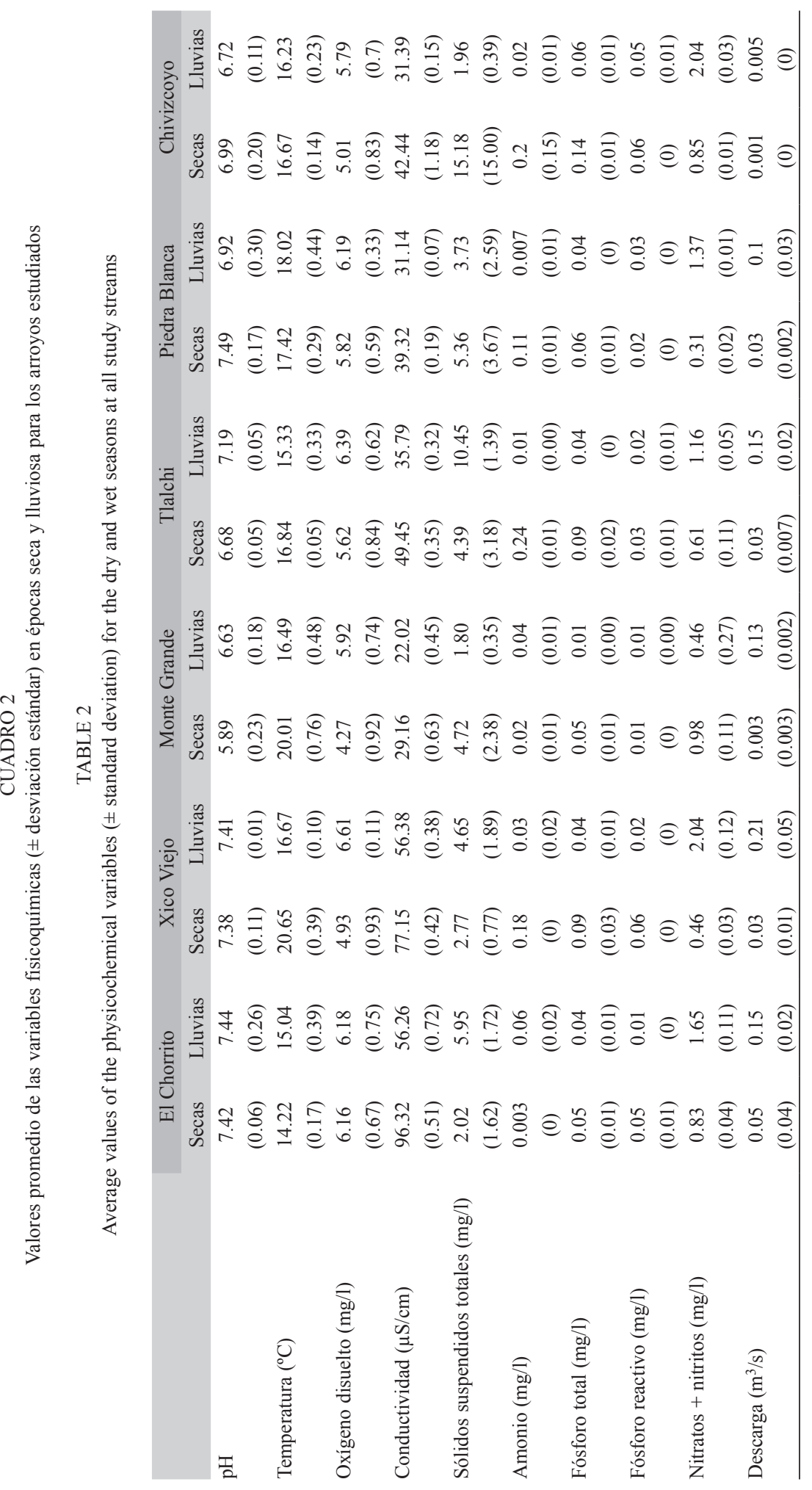




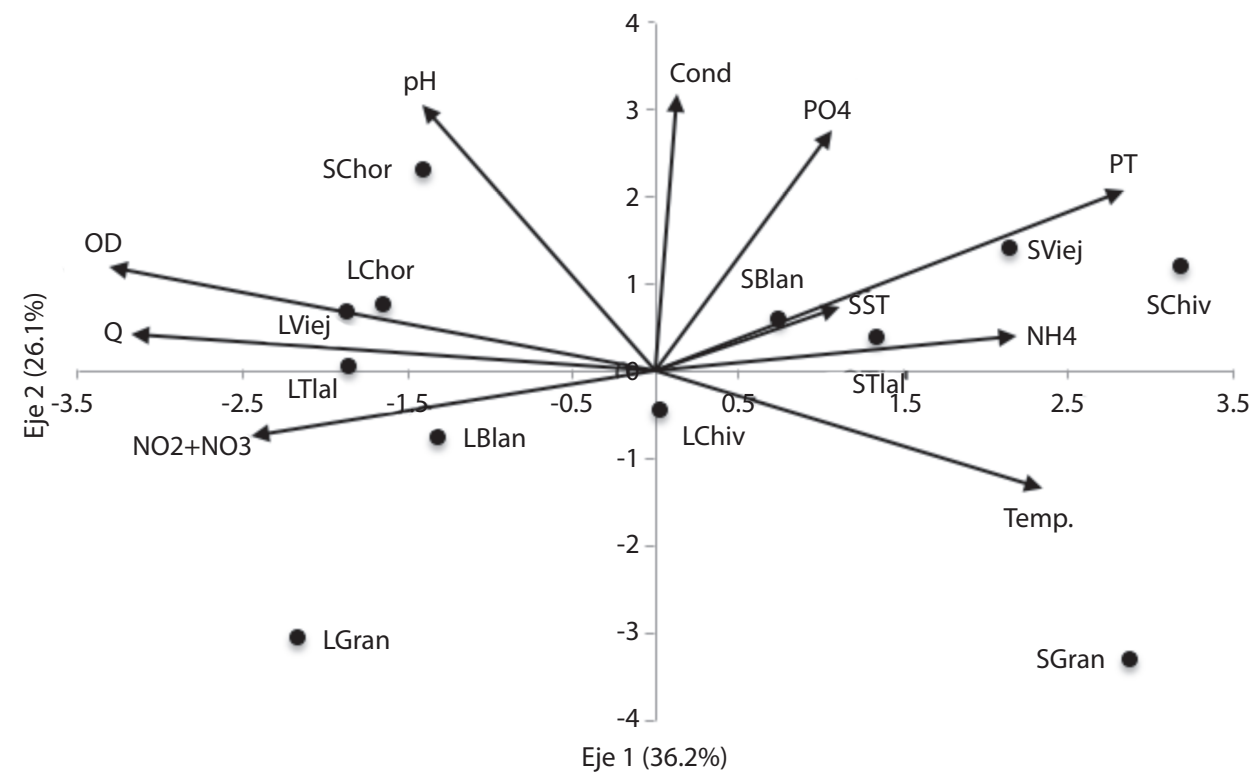

Fig. 1. Distribución de los arroyos de acuerdo a los dos primeros ejes del ACP y el aporte de los parámetros físicoquímicos a estos ejes. Gran: Monte Grande, Blan: Piedra Blanca, Chor: El Chorrito, Tlal: Tlalchi, Viej: Xico Viejo, Chiv: Chivizcoyo. S: época seca L: época lluviosa.

Fig. 1. Stream distribution according to the first two PCA axes and the weight of physicochemical parameters along each axis. Gran: Monte Grande, Blan: Piedra Blanca, Chor: El Chorrito, Tlal: Tlalchi, Viej: Xico Viejo, Chiv: Chivizcoyo. S: dry season L: wet season.

coincidiendo con un gradiente de menor a mayor grado de conservación de la vegetación boscosa (Cuadro 1). En cuanto al agrupamiento de los arroyos en la época seca en relación al eje 2 , no se registró ninguna agrupación relacionada al porcentaje de cobertura del bosque.

Descomposición de hojarasca: La mayoría de los paquetes de hojarasca presentaron poco cambio en su peso inicial en los primeros siete días de sumergidos (menos del 15\%). En este mismo periodo, algunos paquetes en el Chorrito y Xico Viejo en época lluviosa, perdieron más del $40 \%$ de su peso. Posteriormente, el porcentaje de peso perdido por los paquetes fue más variable. Para la última fecha de recolección (35 días), los paquetes habían perdido entre el 30 y el $85 \%$ de su peso inicial. A los 28 días de sumergidos los paquetes, el mayor valor de peso seco libre de cenizas para la época seca se registró en Piedra Blanca (94\%), mientras que para la época lluviosa se determinó en Tlalchi (75\%). Los valores más bajos del peso seco fueron los de Monte Grande (56\%) en época seca y El Chorrito (4.6\%) en época lluviosa. No hubo diferencias significativas entre el peso seco libre de ceniza restante de los arroyos entre épocas $(H=0.02, p=0.88)$.

Las regresiones para calcular las tasas de descomposición $(k)$ fueron significativas para la mayoría de los arroyos en las épocas seca y lluviosa, con excepción de Xico Viejo en ambas épocas y Tlalchi en la época seca (Cuadro 3). Para la época seca, Monte Grande presentó el mayor valor de $k(0.041)$, mientras que para la época lluviosa fue El Chorrito (0.096). En cuanto a los valores más bajos, estos fueron los de Piedra Blanca en época seca $(k=0.01)$ y para la época lluviosa fueron Piedra Blanca y Chivizcoyo $(k=0.014)$, que son los arroyos con la mayor cobertura boscosa en su área de captación (Cuadro 1). No hubo diferencias significativas entre las tasas de descomposición de los arroyos entre épocas $(H=0.09$, 
CUADRO 3

Valores de tasa de descomposición para paquetes de hojarasca de Haya (P. mexicana)

TABLE 3

Breakdown rate values for leaf packs of Sycamore (P. mexicana)

\begin{tabular}{lcccccc}
\multicolumn{1}{c}{ Arroyo } & Época & $\mathrm{n}$ & $k$ & $r^{2}$ & $p$ & pslc (\%) \\
El Chorrito & Seca & 15 & 0.015 & 0.40 & 0.008 & 58.4 \\
& Lluvia & 7 & 0.096 & 0.79 & 0.003 & 4.6 \\
Xico Viejo & Seca & 10 & 0.012 & 0.21 & 0.158 & 74.6 \\
& Lluvia & 11 & 0.007 & 0.02 & 0.676 & 73.1 \\
Monte Grande & Seca & 11 & 0.041 & 0.52 & 0.007 & 56.3 \\
& Lluvia & 11 & 0.027 & 0.47 & 0.014 & 72.8 \\
Tlalchi & Seca & 6 & 0.013 & 0.51 & 0.071 & 65.8 \\
\multirow{2}{*}{ Piedra Blanca } & Lluvia & 11 & 0.015 & 0.55 & 0.005 & 75 \\
\multirow{3}{*}{ Chivizcoyo } & Seca & 14 & 0.01 & 0.36 & 0.017 & 94 \\
& Lluvia & 14 & 0.014 & 0.60 & 0.0006 & 72.9 \\
\hline
\end{tabular}

Valores de tasa de descomposición $(k)$, coeficiente de determinación $\left(r^{2}\right)$ y sus respectivas significancias $(p)$ para los paquetes de hojarasca (n) obtenidos mediante regresión lineal para 6 arroyos de la cuenca alta del río La Antigua en épocas seca y lluviosa. También se incluye el peso seco libre de cenizas (pslc) después de 28 días. En Chivizcoyo no se midió la descomposición durante la época seca.

$p=0.76$ ). Los arroyos con regresiones no significativas (Tlalchi en época seca y Xico Viejo en ambas épocas) no se tomaron en cuenta para posteriores análisis.

Insectos acuáticos: Se recolectó un total de 4017 insectos, 3207 (en 57 paquetes de hojarasca) para la época seca y 810 (en 74 paquetes de hojarasca) para la época lluviosa. En todos los paquetes de hojarasca se encontraron 42 géneros, 35 para la época seca y 28 para la lluviosa (Cuadro 4). Los dos géneros con la mayor abundancia relativa para la época seca fueron Lepidostoma (Trichoptera) con $14.6 \%$ del total de individuos en época seca y Cernotina (Trichoptera; 2.8\%). Para la época lluviosa Simulium (Diptera; 11.8\% del total de individuos) y Phylloicus (Trichoptera; 9.5\%) fueron los más abundantes. Aunque no se realizó la determinación a género de la familia Chironomidae, este fue el grupo más abundante en ambas épocas, con el $73.5 \%$ de la abundancia para la época seca y $51.7 \%$ para la lluviosa.

El análisis de agrupamiento para la época seca mostró valores bajos de similitud (Fig. 2), la mayor similitud se dio para El Chorrito y Tlalchi ( $\mathrm{J}=0.46$, grupo 1) seguidos por la similitud entre Monte Grande y Piedra Blanca $(\mathrm{J}=0.45$, grupo 3 ). Xico Viejo fue el arroyo con la composición de insectos más diferente, su composición fue más cercana a la de El Chorrito y Tlalchi que a la de los demás arroyos. En cuanto a la época lluviosa el grupo 1, formado por los arroyos con la mayor cobertura boscosa (Piedra Blanca y Chivizcoyo), presentó la mayor similitud $(\mathrm{J}=0.52)$, seguidos por el grupo de dos arroyos (grupo 4) con porcentaje de cobertura cercana entre ellos (Monte Grande y Xico Viejo, J=0.44). En cuanto a Tlalchi (grupo 2) y El Chorrito (grupo 3) presentaron valores de similitud bajos con todos los arroyos para la época lluviosa.

Los grupos funcionales encontrados en los paquetes de hojarasca fueron fragmentadores, raspadores, recolectores y depredadores. Para ambas épocas el grupo funcional con la mayor abundancia relativa fue el de los recolectores (78.8\% para la época seca y $75.4 \%$ para la época lluviosa), seguido por los fragmentadores $(16.3 \%$ para la época seca y $19.3 \%$ para la 


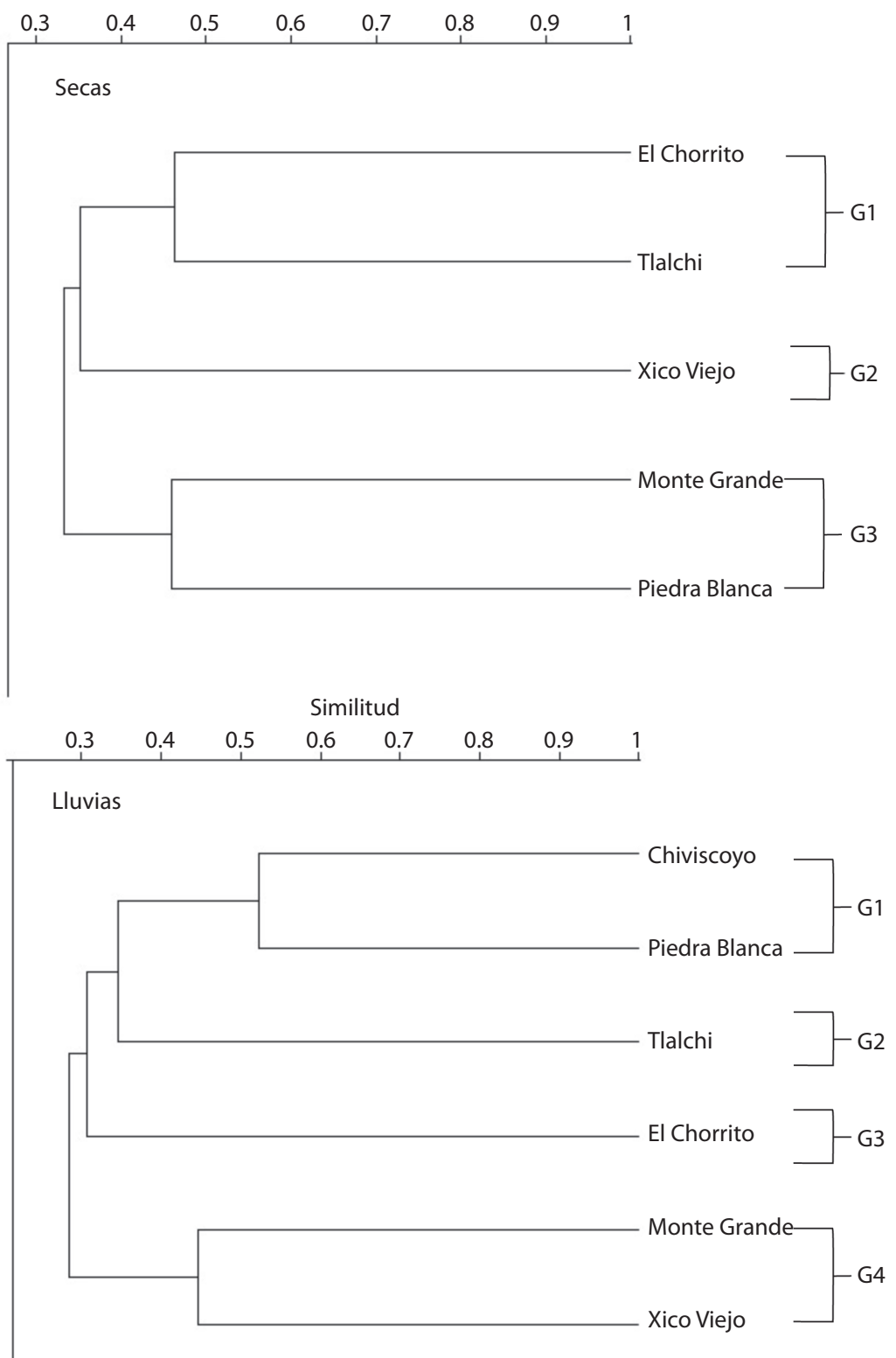

Fig. 2. Análisis de agrupamiento, utilizando el índice de Jaccard, para los arroyos en época seca y lluviosa, se utilizaron todos los géneros y Chironomidae. G 1-4: Grupos 1-4. En Chivizcoyo no se midió la descomposición durante la época seca. Fig. 2. Cluster analysis, using Jaccard index, for the streams during dry and wet seasons, all genera and Chironomidae were used. G 1-4: Groups 1-4. Leaf litter decomposition was not measured in Chivizcoyo during the dry season. 
lluviosa) (Fig. 3). Al comparar las abundancias de cada arroyo en las dos épocas, en general se encontraron más recolectores y raspadores
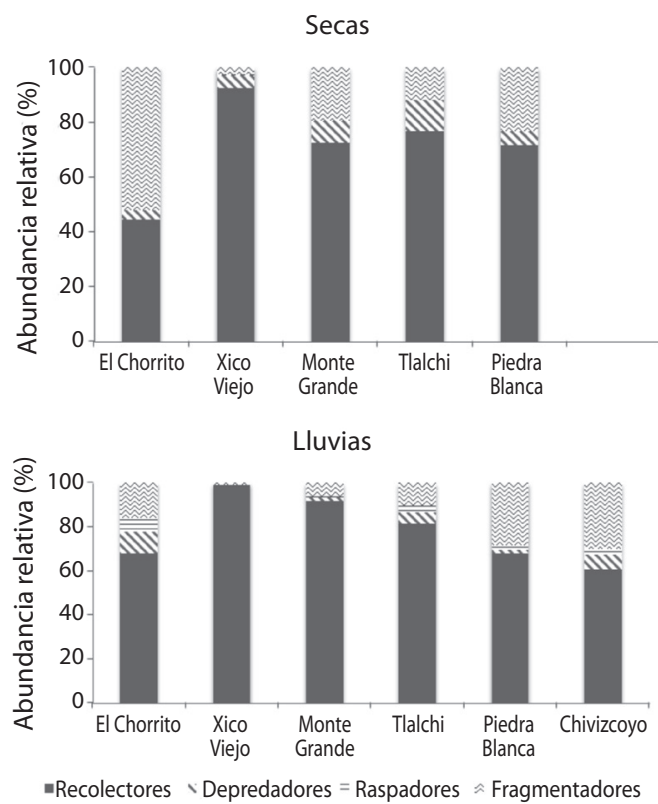

Fig. 3. Abundancia relativa de los grupos funcionales encontrados en los paquetes de hojarasca en los arroyos muestreados para las épocas seca y lluviosa. En Chivizcoyo no se midió la descomposición durante la época seca.

Fig. 3. Relative abundances of functional feeding groups found in the leaf packs at all study streams during dry and wet seasons. Leaf litter decomposition was not measured in Chivizcoyo during the dry season. en la época lluviosa, y más depredadores y fragmentadores en la época seca. Piedra Blanca presentó el patrón opuesto para recolectores, depredadores y fragmentadores. En la época seca El Chorrito fue el único arroyo con el grupo funcional de fragmentadores más abundante que los recolectores (Fig. 3).

Relación entre la tasa de descomposición, porcentaje de bosque, los insectos acuáticos y la fisicoquímica del agua: Al realizar la regresión múltiple (stepwise) incluyendo la descarga, PT, temperatura, $\mathrm{NH}_{4}{ }^{+}$, el porcentaje de bosque en el área de captación, y la cantidad de insectos fragmentadores y no fragmentadores, solo el porcentaje de bosque y la cantidad de insectos no fragmentadores entraron en el modelo de regresión (modelo completo: $r^{2}$ ajustado=0.58). De estas dos variables, solo el porcentaje de bosque fue significativo $(p=0.04)$ y mostró una relación negativa con $k$ (Fig. 4). La regresión múltiple para el peso seco libre de ceniza restante en el día 28 , utilizando las variables usadas en la regresión para $k$ (descarga, $\mathrm{PT}$, temperatura, $\mathrm{NH}_{4}^{+}$, el porcentaje de bosque en el área de captación, y la cantidad de insectos fragmentadores y no fragmentadores), no fue significativa $\left(r^{2}\right.$ ajustado $\left.=0.34\right)$.

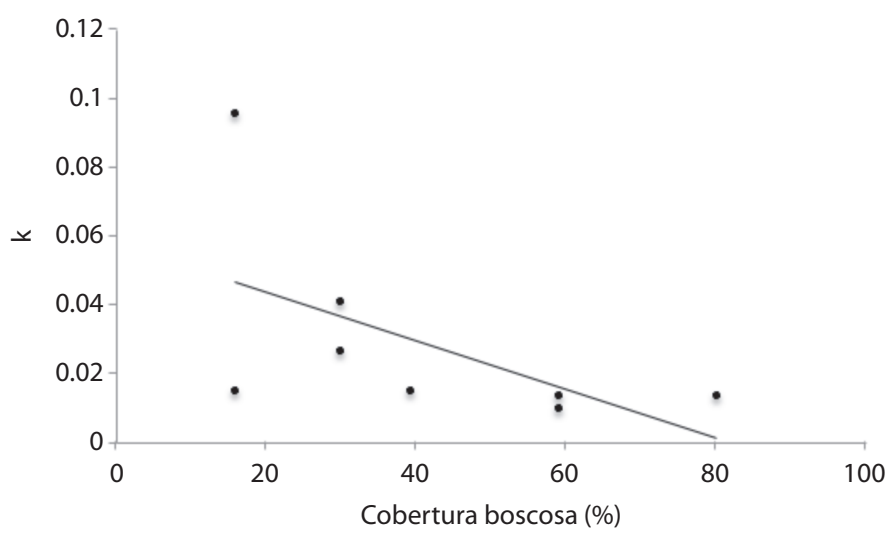

Fig. 4. Relación de la tasa de descomposición $(k)$ y el porcentaje de cobertura boscosa en el área de captación para los arroyos muestreados, se incluyen las $k$ de las épocas seca y lluviosa.

Fig. 4. Relation between breakdown rate $(k)$ and percent forest in the catchment at all study streams, including $k$ values for dry and wet seasons. 
CUADRO 4

Valores de abundancia relativa para los taxa encontrados en los paquetes de hojarasca en los seis arroyos durante las épocas seca y lluviosa

TABLE 4

Relative abundance values for taxa found in leaf packs at the six study streams during dry and wet seasons

\begin{tabular}{|c|c|c|c|c|c|c|c|c|c|c|c|}
\hline \multirow{2}{*}{ Taxon } & \multicolumn{2}{|c|}{ El Chorrito } & \multicolumn{2}{|c|}{ Xico Viejo } & \multicolumn{2}{|c|}{ Monte Grande } & \multicolumn{2}{|c|}{ Tlalchi } & \multicolumn{2}{|c|}{ Piedra Blanca } & \multirow{2}{*}{$\begin{array}{c}\text { Chivizcoyo } \\
\text { Lluvias }\end{array}$} \\
\hline & Secas & Lluvias & Secas & Lluvias & Secas & Lluvias & Secas & Lluvias & Secas & Lluvias & \\
\hline \multicolumn{12}{|l|}{ Ephemeroptera } \\
\hline \multicolumn{12}{|l|}{ Baetidae } \\
\hline Cloeodes & 0 & 0 & 0 & 0 & 1.79 & 0 & 0 & 0 & 0.50 & 0 & 0 \\
\hline Fallceon & 0 & 25.81 & 0.15 & 2.88 & 0 & 0.63 & 0 & 3.39 & 0 & 7.49 & 8.70 \\
\hline \multicolumn{12}{|l|}{ Leptohyphidae } \\
\hline Leptohyphes & 0 & 0 & 0.05 & 0 & 0 & 0.63 & 0 & 0 & 0 & 0 & 0 \\
\hline Tricorythodes & 0.59 & 3.23 & 0.15 & 0 & 0.89 & 0 & 1.67 & 0 & 0.25 & 0.44 & 0.87 \\
\hline \multicolumn{12}{|l|}{ Leptophlebiidae } \\
\hline Farrodes & 1.03 & 0 & 2.91 & 0 & 8.93 & 0 & 3.33 & 0 & 3.53 & 5.29 & 3.91 \\
\hline Neochoroterpes & 0 & 0 & 0.15 & 0 & 0.89 & 0 & 0 & 0 & 0.25 & 0 & 0.43 \\
\hline \multicolumn{12}{|l|}{ Odonata } \\
\hline \multicolumn{12}{|l|}{ Aeshnidae } \\
\hline Oplonaeschna & 0.15 & 0 & 0 & 0 & 0 & 0 & 0 & 0 & 0.25 & 0 & 0 \\
\hline \multicolumn{12}{|l|}{ Calopterygidae } \\
\hline Hetaerina & 0.74 & 0 & 0.05 & 0 & 2.68 & 1.26 & 10 & 1.69 & 1.01 & 0 & 1.30 \\
\hline \multicolumn{12}{|l|}{ Coenagrionidae } \\
\hline Argia & 0 & 0 & 0 & 0 & 1.79 & 0 & 0 & 0 & 0 & 0 & 0 \\
\hline \multicolumn{12}{|l|}{ Lestidae } \\
\hline Archilestes & 0 & 0 & 0 & 0 & 0.89 & 0 & 0 & 0 & 0 & 0 & 0 \\
\hline \multicolumn{12}{|l|}{ Plecoptera } \\
\hline \multicolumn{12}{|l|}{ Nemouridae } \\
\hline Amphinemura & 0.74 & 16.13 & 0.10 & 0.96 & 0 & 3.14 & 0 & 10.17 & 10.58 & 12.78 & 0 \\
\hline \multicolumn{12}{|l|}{ Perlidae } \\
\hline Anacroneuria & 0 & 3.23 & 0 & 0 & 0 & 0 & 0 & 1.69 & 0.25 & 0.44 & 4.35 \\
\hline \multicolumn{12}{|l|}{ Hemiptera } \\
\hline \multicolumn{12}{|l|}{ Belostomatidae } \\
\hline Abedus & 0 & 0 & 0.15 & 0 & 0 & 0 & 0 & 0 & 0 & 0 & 0 \\
\hline \multicolumn{12}{|l|}{ Naucoridae } \\
\hline Ambrysus & 0 & 0 & 0.10 & 0 & 0 & 0 & 0 & 0 & 0 & 0 & 0 \\
\hline Coleoptera & & & & & & & & & & & \\
\hline Elmidae & & & & & & & & & & & \\
\hline Ancyronyx & 0 & 3.23 & 0 & 0 & 0 & 0 & 0 & 0 & 0 & 0 & 0 \\
\hline Cylloepus & 0 & 0 & 0 & 0 & 0 & 0 & 0 & 0 & 0 & 0 & 0.43 \\
\hline Heterelmis & 0.15 & 0 & 0.56 & 0 & 0.89 & 0 & 0 & 10.17 & 0.50 & 1.32 & 0.87 \\
\hline Microcylloepus & 0 & 0 & 0.46 & 0 & 0 & 0 & 1.67 & 0 & 0 & 0 & 0 \\
\hline Neoelmis & 0 & 6.45 & 0.05 & 0 & 0 & 0 & 0 & 1.69 & 0.25 & 2.20 & 1.30 \\
\hline Rhizelmis & 0 & 0 & 0.05 & 0 & 0 & 0.63 & 0 & 0 & 0 & 0 & 0.43 \\
\hline Hydraenidae & & & & & & & & & & & \\
\hline Ochthebius & 0 & 0 & 0 & 0 & 0 & 0 & 0 & 0 & 0.50 & 0 & 0 \\
\hline Hydrophilidae & & & & & & & & & & & \\
\hline Laccobius & 0 & 0 & 0 & 0 & 0 & 0 & 0 & 0 & 0.25 & 0 & 0 \\
\hline
\end{tabular}


CUADRO 4 (Continuación) / TABLE 4 (Continued)

\begin{tabular}{|c|c|c|c|c|c|c|c|c|c|c|c|}
\hline \multirow{2}{*}{ Taxon } & \multicolumn{2}{|c|}{ El Chorrito } & \multicolumn{2}{|c|}{ Xico Viejo } & \multicolumn{2}{|c|}{ Monte Grande } & \multicolumn{2}{|c|}{ Tlalchi } & \multicolumn{2}{|c|}{ Piedra Blanca } & \multirow{2}{*}{$\begin{array}{c}\text { Chivizcoyo } \\
\text { Lluvias }\end{array}$} \\
\hline & Secas & Lluvias & Secas & Lluvias & Secas & Lluvias & Secas & Lluvias & Secas & Lluvias & \\
\hline \multicolumn{12}{|l|}{ Psephenidae } \\
\hline Psephenus & 0 & 0 & 0.56 & 0 & 0 & 0 & 0 & 0 & 0 & 0 & 0 \\
\hline \multicolumn{12}{|l|}{ Trichoptera } \\
\hline \multicolumn{12}{|l|}{ Calamoceratidae } \\
\hline Phylloicus & 0 & 0 & 0 & 0 & 3.57 & 2.52 & 0 & 0 & 0.25 & 6.61 & 25.22 \\
\hline \multicolumn{12}{|l|}{ Helicopsychidae } \\
\hline Helicopsyche & 0 & 0 & 0.10 & 0 & 0 & 0 & 0 & 0 & 0 & 0 & 0.43 \\
\hline \multicolumn{12}{|l|}{ Hydrobiosidae } \\
\hline Atopsyche & 0 & 0 & 0.10 & 0 & 0 & 0 & 0 & 0 & 0.25 & 0.44 & 0 \\
\hline \multicolumn{12}{|l|}{ Hydropsychidae } \\
\hline Leptonema & 0 & 0 & 0 & 0 & 0 & 0 & 0 & 3.39 & 0 & 0 & 0 \\
\hline \multicolumn{12}{|l|}{ Hydroptilidae } \\
\hline Ithytrichia & 0 & 0 & 0 & 0 & 0 & 0 & 0 & 1.69 & 0 & 0 & 0 \\
\hline Neotrichia & 0 & 0 & 0 & 0 & 0 & 0 & 0 & 0 & 0 & 0 & 0.43 \\
\hline Ochrotrichia & 0 & 0 & 0.15 & 0 & 0 & 0 & 0 & 0 & 0 & 0 & 0 \\
\hline \multicolumn{12}{|l|}{ Lepidostomatidae } \\
\hline Lepidostoma & 51.55 & 0 & 2.30 & 0 & 16.07 & 0.63 & 11.67 & 0 & 12.34 & 9.25 & 2.61 \\
\hline \multicolumn{12}{|l|}{ Leptoceridae } \\
\hline Nectopsyche & 0 & 0 & 0 & 0 & 0 & 0 & 0 & 0 & 0 & 0 & 2.17 \\
\hline Oecetis & 0 & 0 & 0.10 & 0 & 0 & 0 & 0 & 1.69 & 0 & 0 & 0.43 \\
\hline \multicolumn{12}{|c|}{ Polycentropodidae } \\
\hline Cernotina & 1.77 & 0 & 3.47 & 0 & 2.68 & 0 & 0 & 0 & 1.51 & 0 & 0 \\
\hline \multicolumn{12}{|c|}{ Xiphocentronidae } \\
\hline Xiphocentron & 0 & 0 & 0 & 0 & 0 & 0 & 0 & 1.69 & 0 & 0 & 0 \\
\hline \multicolumn{12}{|l|}{ Diptera } \\
\hline \multicolumn{12}{|l|}{ Athericidae } \\
\hline Suragina & 0.29 & 6.45 & 0.10 & 0 & 0 & 0 & 1.67 & 0 & 0 & 0 & 0 \\
\hline \multicolumn{12}{|l|}{ Ceratopogonidae } \\
\hline Bezzia & 0.15 & 0 & 0.26 & 0 & 0 & 0 & 0 & 0 & 0 & 0 & 0.43 \\
\hline Chironomidae & 42.42 & 35.48 & 87.45 & 89.42 & 58.04 & 37.74 & 70 & 57.63 & 62.47 & 52.42 & 44.35 \\
\hline \multicolumn{12}{|l|}{ Dixidae } \\
\hline Dixa & 0.44 & 0 & 0.41 & 0 & 0 & 0 & 0 & 0 & 3.27 & 0.44 & 0.43 \\
\hline Empididae & & & & & & & & & & & \\
\hline Neoplasta & 0 & 0 & 0 & 0 & 0 & 0 & 0 & 0 & 0.50 & 0.44 & 0.43 \\
\hline Muscidae & & & & & & & & & & & \\
\hline Limnophora & 0 & 0 & 0 & 0 & 0 & 0 & 0 & 0 & 0.25 & 0 & 0 \\
\hline Simuliidae & & & & & & & & & & & \\
\hline Simulium & 0 & 0 & 0 & 6.73 & 0.89 & 52.83 & 0 & 5.08 & 0.76 & 0.44 & 0.43 \\
\hline Tipulidae & & & & & & & & & & & \\
\hline Dicranota & 0 & 0 & 0.05 & 0 & 0 & 0 & 0 & 0 & 0.25 & 0 & 0 \\
\hline Total & 100 & 100 & 100 & 100 & 100 & 100 & 100 & 100 & 100 & 100 & 100 \\
\hline
\end{tabular}




\section{DISCUSIÓN}

Los arroyos muestreados presentaron un amplio gradiente de cobertura boscosa, el cual se vio reflejado en la fisicoquímica del agua, como lo muestra el ACP. El efecto de la vegetación ribereña sobre los parámetros fisicoquímicos del agua ha sido documentado en otras áreas templadas y tropicales (Close \& DaviesColley, 1990; Heartsill-Scalley \& Aide, 2003). De igual forma hubo una diferenciación de los arroyos entre las épocas, debida principalmente a la descarga, nutrientes, temperatura y OD. Esto concuerda con lo reportado por Ramírez, Pringle \& Douglas (2006), donde los cambios estacionales en los parámetros físicoquímicos mostraron relación con la descarga. En cuanto a los cambios en los parámetros fisicoquímicos relacionados con la cobertura boscosa y las épocas, Johnson, Richards, Host \& Arthur (1997) encontraron una relación similar a la encontrada en nuestro estudio, donde los mayores cambios entre épocas se mostraron en nitrógeno, y entre usos de suelo en el $\mathrm{pH}$.

En todos los arroyos la tasa de descomposición de las hojas de Platanus mexicana tuvo valores mayores a 0.01 , y de acuerdo a las categorías establecidas por Petersen \& Cummins (1974) las tasas de descomposición obtenidas fueron rápidas. Estos valores se encuentran dentro de los rangos reportados por Swan \& Palmer (2004) en un estudio realizado en un arroyo de zona templada, durante el verano, con seis especies de plantas, dentro de las que se incluye una especie de Platanus. A pesar de que las tasas de descomposición aquí reportadas son catalogadas como rápidas, existen hojas con tasa de descomposición mayor (LeRoy \& Marks, 2006). Mathuriau \& Chauvet (2002) para un arroyo tropical, atribuyen la rápida tasa de descomposición de la hojarasca a la relativamente alta y estable temperatura del agua (entre 18 y $27.5^{\circ} \mathrm{C}$ ) comparada con zonas templadas. Benstead (1996) también señala a la temperatura del agua como un posible factor importante en las rápidas tasas de descomposición encontradas en un arroyo tropical. De acuerdo a lo anterior la temperatura de los arroyos en este estudio fue estable (14.2 a $\left.20.6^{\circ} \mathrm{C}\right)$, a pesar de ser un parámetro importante en la diferenciación fisicoquímica de los arroyos entre épocas. Esta estabilidad en la temperatura podría explicar los altos valores obtenidos para la tasa de descomposición. Por otra parte, en este estudio no se encontraron diferencias en la tasa de descomposición de P. mexicana entre épocas. Se ha reportado a la temperatura como uno de los principales factores que influyen en la tasa de descomposición en zonas templadas. Swan \& Palmer (2004) señalan como el principal factor causante de cambios en la tasa de descomposición, entre verano y otoño, a la temperatura del agua que cambia de $22^{\circ} \mathrm{C}$ en verano a $4.5^{\circ} \mathrm{C}$ en otoño. En contraste, en este estudio al encontrarse los arroyos en una zona tropical, el intervalo de temperatura entre épocas fue pequeño (época seca: 14.2 a $20.6^{\circ} \mathrm{C}$, época lluviosa: 15 a $17.4^{\circ} \mathrm{C}$ ). Otra explicación para no haber encontrado diferencias en la tasa de descomposición entre épocas, podría ser que esta se ve afectada por distintos factores, que varían en el tiempo, como la velocidad de colonización por los macroinvertebrados y las características fisicoquímicas del agua, entre otros. Para las dos épocas el efecto de cada factor sobre la descomposición puede ser distinto sin que esto se vea reflejado en la tasa de descomposición debido a que en conjunto el efecto de los factores es igual para las dos épocas. Por ejemplo, en época seca el factor dominante de la descomposición podría ser la mayor abundancia de los insectos acuáticos mientras para la época lluviosa podría ser la alta variabilidad del flujo de agua.

La mayor abundancia de los insectos en la época seca coincide con lo observado en otros estudios (Miller \& Golladay, 1996; Braioni, Salmoiraghi, Gumiero \& Cisotto, 1997). La gran diferencia en las abundancias se puede atribuir a dos factores principales. Primero, a finales de la época seca es el momento en que emergen muchos de los estados adultos de los insectos acuáticos como parte de una estrategia para evitar crecientes (Lytle, 2000; 2002), por lo que para la época lluviosa (que ocurre después de la época seca y antes de la época de 
nortes) hay pocos individuos o los que pudieran abundar son de tamaño pequeño. Segundo, las fuertes corrientes durante la época lluviosa, que incluso ocasionan deslaves, pueden causar arrastre de la fauna acuática. Miller \& Golladay (1996) mostraron que hay una pérdida de más del $90 \%$ de la densidad de insectos después de un aluvión. Esto es respaldado al observar que Simulium fue el género más abundante durante la época lluviosa. Se ha encontrado que este género aumenta su abundancia después de perturbaciones del sustrato en la época lluviosa, mientras que la de los demás macroinvertebrados disminuye (Rosser \& Pearson, 1995). La mayor abundancia relativa de Chironomidae en todos los arroyos, con excepción de Monte Grande en época lluviosa y El Chorrito en época seca, concuerda con lo encontrado en otros trabajos (Menéndez, Hernández \& Comín, 2003; Li, Ng \& Dudgeon, 2009; Guevara, 2011). Lepidostoma fue el género más abundante en la mayoría de los arroyos en época seca y esto pudo deberse a que las condiciones presentes en esta época (como la baja cantidad de OD o la baja descarga), estén favoreciendo la colonización de la hojarasca por parte de este género.

La similitud en la composición de insectos no mostró grupos compartidos entre las dos épocas, según los análisis de agrupamiento. Para la época lluviosa, los grupos con los mayores valores de similitud fueron para arroyos con porcentajes de cobertura boscosa cercanos (Piedra Blanca con Chivizcoyo y Monte Grande con Xico Viejo) pero esto no sucedió para la época seca, haciendo la salvedad que Chivizcoyo no se muestreó en la época seca. Esto podría deberse a que en la época seca la descarga es menor, comparada con la época lluviosa, los paquetes de hojarasca estarían más accesibles para los insectos acuáticos y podrían ser usados por un mayor número de taxa, mientras en la época lluviosa solo podrían ser usados por los insectos capaces de soportar la variabilidad en descarga típica de esta época. Lo anterior concuerda con que se encontraron más géneros e individuos en los paquetes de hojarasca para la época seca y que para la época lluviosa Simulium (género característico de zonas con corriente) fue el género más abundante.

En cuanto a los grupos funcionales, el patrón encontrado, mayor abundancia de recolectores seguido por los fragmentadores, concuerda con lo reportado por otros estudios en el trópico (Mathuriau \& Chauvet, 2002; Ardón, Stallcup \& Pringle, 2006; Moretti, Gonçalves, Ligeiro \& Callisto, 2007; Boyero, Ramírez, Dudgeon \& Pearson, 2009). Esto indica que los fragmentadores están aportando poco a la descomposición de la hojarasca y que tal vez el mayor aprovechamiento de la misma, por los insectos acuáticos, sea como refugio y como fuente indirecta de alimento, al aprovechar la materia orgánica fina acumulada y la biopelícula (Smith, 1986). El uso de la hojarasca por parte de los insectos fue similar en las dos épocas ya que el grupo de los recolectores fue el grupo funcional dominante en ambas épocas. El aumento en la abundancia relativa de recolectores y raspadores para la época lluviosa podría deberse a que los paquetes de hojarasca fueron una fuente de alimento y refugio poco común para la época, debido a las fuertes corrientes que suelen arrastrarla (Braioni et al., 1997).

El proceso de descomposición de hojarasca en los arroyos de BMM estudiados mostró estar relacionado con el porcentaje de cobertura boscosa en el área de captación. La importancia de la vegetación del área de captación en la descomposición de la hojarasca ha sido documentada, encontrándose una relación negativa entre las tasas de descomposición y el porcentaje de tierra sin bosque. Esta relación se atribuye al efecto de otros procesos que influyen indirectamente sobre la descomposición, como el aumento de sólidos suspendidos que llegan por escorrentía al cuerpo de agua y aumentan la fragmentación (Sponseller \& Benfield, 2001). De igual forma, Paul et al. (2006) reportan diferencias en la tasa de descomposición para arroyos presentes en distintos usos de suelo, siendo mayor en arroyos de áreas agrícolas y urbanas que en arroyos suburbanos y de bosque. Sin embargo 
señalan que las causas para la mayor tasa de descomposición en áreas agrícolas y urbanas podrían ser distintas, siendo para la primera producto de una mayor actividad biológica facilitada por el enriquecimiento de nutrientes, mientras para la segunda pudo deberse a la fragmentación física por el flujo del agua. La relación negativa entre cobertura boscosa y la tasa de descomposición de hojarasca en nuestro sitio de estudio podría deberse a que el bosque, al funcionar como una barrera física contra los sedimentos llevados por el escurrimiento del agua de lluvia, esté disminuyendo la cantidad de sedimentos provenientes de la ribera y de esta forma la degradación de la hojarasca que se produce por fricción sería menor (Ferreira et al., 2006; Hagen, Webster \& Benfield, 2006). Esto concuerda con lo reportado por Vázquez, Aké-Castillo \& Favila (2011) para la zona de nuestro estudio, donde los arroyos de pastizales y cafetales presentaron mayores valores de SST comparado con arroyos de bosque.

El proceso de descomposición de hojarasca en arroyos de bosque mesófilo de montaña en el área de estudio aparenta ser estable. A pesar de que hubo una clara diferenciación de los arroyos entre épocas, de acuerdo a los parámetros fisicoquímicos del agua y las abundancias relativas de los insectos acuáticos, estas diferencias no se reflejaron en la tasa de descomposición, lo cual sugiere estabilidad en el proceso de descomposición. Sin embargo, esto no significa que los factores que afectan la descomposición se mantengan constantes entre las épocas. Estudios a lo largo de un gradiente de uso de terreno y de impacto físicoquímico más fuerte podrían ayudar a entender mejor los controles sobre este proceso.

\section{AGRADECIMIENTOS}

A José Antonio Gómez, Javier Tolome y Arlet Fuentes por la ayuda durante la fase de campo. Daniela Cela y Ariadna Martínez por su ayuda en la obtención de los parámetros fisicoquímicos del agua. Rosario Landgrave por la ayuda con el análisis SIG para los arroyos muestreados. Al Consejo Nacional de Ciencia y
Tecnología (CONACyT), por su apoyo a través de la beca doctoral No. 229587 para el primer autor y el proyecto No. 101542.

\section{RESUMEN}

Para muchos arroyos, la hojarasca proveniente de la zona ribereña es la base de las redes tróficas, por lo cual el proceso de descomposición de la hojarasca es de gran importancia para el funcionamiento de estos ecosistemas. Con el fin de conocer los factores que afectan la descomposición de la hojarasca en arroyos del bosque mesófilo de montaña (BMM), se utilizaron paquetes de hojarasca durante 35 días. Seis arroyos con un gradiente de perturbación fueron estudiados en la época seca y lluviosa. Se determinó la relación entre la tasa de descomposición de la hojarasca $(k)$, con algunos parámetros físicoquímicos del cuerpo de agua y los macroinvertebrados colonizadores de los paquetes. Los factores fisicoquímicos mostraron una separación de los cuerpos de agua según la época a través de un Análisis de Componentes Principales (ACP). De los insectos colonizadores de los paquetes de hojarasca, el grupo funcional de los recolectores fue el más abundante, seguido por los fragmentadores. Solo se encontró una relación negativa entre la $k$ y el porcentaje de cobertura boscosa $(p=0.04)$ al realizar una regresión múltiple por pasos en la que se incluyeron algunos parámetros fisicoquímicos, el porcentaje de cobertura boscosa y las abundancias promedio de los insectos fragmentadores y no fragmentadores. La descomposición de la hojarasca en los arroyos estudiados parece ser un proceso estable a lo largo del año, aunque los factores responsables por la regulación pueden diferir en las distintas épocas.

Palabras clave: descomposición hojarasca, insectos acuáticos, cobertura boscosa, grupos funcionales.

\section{REFERENCIAS}

Aldridge, K. T., Brookes, J. D., \& Ganf, G. G. (2009). Rehabilitation of stream ecosystem functions through the reintroduction of coarse particulate organic matter. Restoration Ecology, 17, 97-106.

APHA. (1998). Standard methods for examination of water and wastewater. Washington, DC, USA.

Ardón, M., Stallcup, L. A. \& Pringle, C. M. (2006). Does leaf quality mediate the stimulation of leaf breakdown by phosphorus in Neotropical streams? Freshwater Biology, 51, 618-633.

Barlocher, F. (2005). Leaf mass loss estimated by litter bag technique. In M. A. S. Graca, F. Barlocher \& M. O. Gessner (Eds.), Methods to study litter decomposition: A practical guide (pp. 37-42). Dordrecht: Springer. 
Benstead, J. P. (1996). Macroinvertebrates and the processing of leaf litter in a tropical stream. Biotropica, 28, 367-375.

Biggs, T. W., Dunne, T., \& Martinelli, L. A. (2004). Natural controls and human impacts on stream nutrient concentrations in a deforested region of the brazilian amazon basin. Biogeochemistry, 68, 227-257.

Boyero, L., Pearson, R. G., \& Bastian, M. (2007). How biological diversity influences ecosystem function: a test with a tropical stream detritivore guild. Ecological Research, 22, 551-558.

Boyero, L., Ramírez, A., Dudgeon, D., \& Pearson, R. G. (2009). Are tropical streams really different? Journal of the North American Benthological Society, 28, 397-403.

Braioni, M. G., Salmoiraghi, G., Gumiero, B., \& Cisotto, P. (1997). Breakdown and colonization of alder in regulated italian watercourses. Limnetica, 13, 25-32.

Casas, J., Gessner, M. O., López, D., \& Descals, E. (2011). Leaf-litter colonization and breakdown in relation to stream typology: insights from Mediterranean loworder streams. Freshwater Biology, 56, 2594-2608.

Close, M. E., \& Davies-Colley, R. J. (1990). Baseflow chemistry in New Zealand rivers 2. Influence of environmental factor. New Zealand Journal of Marine and Freshwater Research, 24, 343-356.

Cummins, K. W., Merritt, R. W., \& Andrade, P. C. N. (2005). The use of invertebrate functional groups to characterize ecosystem attributes in selected streams and rivers in south Brazil. Studies on Neotropical Fauna and Environment, 40, 69-89.

Ferreira, V., Graça, M. A. S., de Lima, J. L. M. P., \& Gomes, R. (2006). Role of physical fragmentation and invertebrate activity in the breakdown rate of leaves. Archiv Fur Hydrobiologie, 165, 493-513.

García-Franco, J. G., Castillo-Campos, G., Mehltreter, K., Martínez, M. L., \& Vázquez, G. (2008). Composición florística de un bosque mesófilo del centro de Veracruz, México. Boletín de la Sociedad Botánica de México, 83, 37-52.

Gonçalves, J. F., Rezende, R. D., França, J., \& Callisto, M. (2012). Invertebrate colonization during leaf processing of native, exotic and artificial detritus in a tropical stream. Marine and Freshwater Research $63,428-439$

Graça, M. A. S. (2001). The role of invertebrates on leaf litter decomposition in streams - a review. International Review of Hydrobiology, 86, 383-393.

Graça, M. A. S., Cressa, C., Gessner, M. O., Feio, M. J., Callies, K. A., \& Barrios, C. (2001). Food quality, feeding preferences, survival and growth of shredders from temperate and tropical streams. Freshwater Bio$\log y, 46,947-957$
Guevara, M. (2011). Insectos acuáticos y calidad del agua en la cuenca y embalse del río Peñas Blancas, Costa Rica. Revista de Biología Tropical 59, 635-654.

Hagen, E. M., Webster, J. R., \& Benfield, E. F. (2006). Are leaf breakdown rates a useful measure of stream integrity along an agricultural landuse gradient? Journal of the North American Benthological Society, $25,330-343$

Hauer, F. R., \& Lamberti, G. A. (2007). Methods in stream ecology. San Diego: Academic.

Heartsill-Scalley, T., \& Aide, T. M. (2003). Riparian vegetation and stream condition in a tropical agriculture-secondary forest mosaic. Ecological Applications, $13,225-234$.

Huryn, A. D., Butz-Huryn, V. M., Arbuckle, C. J., \& Tsomides, L. (2002). Catchment land-use, macroinvertebrates and detritus processing in headwater streams: taxonomic richness versus function. Freshwater Biology, 47, 401-415.

INEGI. (2004). Conjunto de datos vectoriales de las cartas topográficas E14B26, E14B27, E14B36, E14B37. Escala 1:50 000.

Johnson, L., Richards, C., Host, G., \& Arthur, J. (1997). Landscape influences on water chemistry in midwestern stream ecosystems. Freshwater Biology, 37, 193-208.

Leroy, C. J., \& Marks, J. C. (2006). Litter quality, stream characteristics and litter diversity influence decomposition rates and macroinvertebrates. Freshwater Biology, 51, 605-617.

Li, A. O. Y., Ng, L. C. Y., \& Dudgeon, D. (2009). Effects of leaf toughness and nitrogen content on litter breakdown and macroinvertebrates in a tropical stream. Aquatic Science, 71, 80-93.

Lytle, D. A. (2000). Biotic and abiotic effects of flash flooding in a montane desert stream. Archiv für Hydrobiologie, 150, 85-100.

Lytle, D. A. (2002). Flash floods and aquatic insect lifehistory evolution: evaluation of multiple models. Ecology, 83, 370-385.

Martínez, M. (1994). Catálogo de nombres vulgares y cientificos de plantas mexicanas. Ciudad de México, México: Fondo de Cultura Económica.

Martínez, M. L., Pérez-Maqueo, O., Vázquez, G., CastilloCampos, G., García-Franco, J., Mehltreter, K., Equihua, M., \& Landgrave, R. (2009). Effects of land use change on biodiversity and ecosystem services in tropical montane cloud forests of Mexico. Forest Ecology and Management, 258, 1856-1863.

Mathuriau, C., \& Chauvet, E. (2002). Breakdown of leaf litter in a neotropical stream. Journal of the North American Benthological Society, 21, 384-396. 
Menéndez, M., Hernández, O., \& Comín, F. A. (2003). Seasonal comparisons of leaf processing rates in two Mediterranean rivers with different nutrient availability. Hydrobiologia, 495, 159-169.

Merritt, R. W., \& Cummins, K. W. (1996). An Introduction to the Aquatic Insects of North America. Iowa, EEUU: Kendall/Hunt.

Miller, A. M., \& Golladay, S. W. (1996). Effects of spates and drying on macroinvertebrate assemblages of an intermittent and a perennial prairie stream. Journal of the North American Benthological Society, 15, 670-689.

Moretti, M. S., Gonçalves, J. F., Ligeiro, R., \& Callisto, M. (2007). Invertebrates colonization on native tree leaves in a neotropical stream (Brazil). International Review of Hydrobiology, 92, 199-210.

Muñoz-Villers, L. E., \& López-Blanco, J. (2008). Land use/cover changes using landsat TM/ETM images in a tropical and biodiverse mountainous area of centraleastern Mexico. International Journal of Remote Sensing, 29, 71-93.

Navarrete-Vázquez, S. I. (2008). Efecto del uso del suelo sobre los peces de la parte alta de la cuenca del río La Antigua, Veracruz. (Tesis de Maestría). Universidad Autónoma de Querétaro, Querétaro, México.

Niu, S. Q., \& Dudgeon, D. (2011). The influence of flow and season upon leaf-litter breakdown in monsoonal Hong Kong streams. Hydrobiologia, 663, 205-215.

Paul, M. J., Meyer, J. L., \& Couch, C. A. (2006). Leaf breakdown in streams differing in catchment land use. Freshwater Biology, 51, 1684-1695.

Pereyra, D., Cruz, D. G., \& Pérez, J. A. A. (2011). La evapotranspiración real (ETR) en la cuenca del río La Antigua, Veracruz: estado actual y ante escenarios de cambio climático. Investigaciones Geográficas, Boletín del Instituto de Geografia, UNAM, 75, 37-50.

Pérez, J., Menéndez, M., Larrañaga, S., \& Pozo, J. (2011). Inter- and intra-regional variability of leaf litter breakdown in reference headwater streams of northern spain: atlantic versus mediterranean streams. International Review of Hydrobiology, 96, 105-117.

Petersen, R. C., \& Cummins, K. W. (1974). Leaf processing in a woodland stream. Freshwater Biology, 4, 343-368.
Ramírez, A., Pringle, C. M., \& Douglas, M. (2006). Temporal and spatial patterns in stream physicochemistry and insect assemblages in tropical lowland streams. Journal of the North American Benthological Society, 25, 108-125.

Rosser, Z. C., \& Pearson, R. G. (1995). Responses of rock fauna to physical disturbance in two Australian tropical rainforest streams. Journal of the North American Benthological Society, 14, 183-196.

Smith, D. L. (1986). Leaf litter processing and the associated invertebrate fauna in a tallgrass prairie stream. American Midland Naturalist, 116, 78-86.

Sponseller, R. A., \& Benfield, E. F. (2001). Influences of land use on leaf breakdown in southern Appalachian headwater streams: a multiple-scale analysis. Journal of the North American Benthological Society, 20, 44-59.

Swan, C. M., \& Palmer, M. A. (2004). Leaf diversity alters litter breakdown in a piedmont stream. Journal of the North American Benthological Society, 23, 15-28.

Vázquez, G., Aké-Castillo, J. A., \& Favila, M. E. (2011). Algal assemblages and their relationship with water quality in tropical mexican streams with different land uses. Hydrobiologia, 667, 173-189.

Wallace, J. B., Eggert, S. L., Meyer, J. L., \& Webster, J. R. (1997). Multiple trophic levels of a forest stream linked to terrestrial litter inputs. Science, 277, 102-104.

Webster, J. R., \& Benfield, E. F. (1986). Vascular plant breakdown in freshwater ecosystems. Annual Review of Ecology and Systematics, 17, 567-594.

Williams-Linera, G. (1993). Bordes de bosque nublado en el parque ecológico Clavijero, Xalapa, Veracruz, México. Revista de Biología Tropical, 41, 107-117.

Williams-Linera, G., Manson, R. H., \& Isunza, E. (2002). La fragmentación del bosque mesófilo de montaña y patrones de uso del suelo en la región oeste de Xalapa, Veracruz, México. Madera y Bosques 8, 73-89.

Yoshimura, M. (2012). Effects of forest disturbances on aquatic insect assemblages. Entomological Science $15,145-154$. 
